\title{
REVISTAS CIENTÍFICAS ESPAÑOLAS DE INFORMACIÓN Y DOCUMENTACIÓN: ANÁLISIS TEMÁTICO Y METODOLÓGICO
}

\section{Library and information science journals in Spain: Thematic and methodological analysis}

Javier Guallar, Núria Ferran-Ferrer, Ernest Abadal y Adán Server

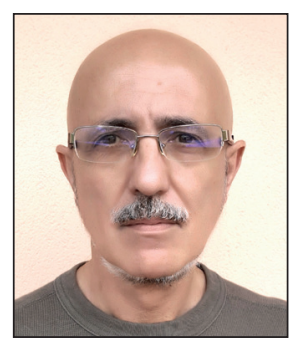

Javier Guallar, doctor en Información y Documentación, es profesor del Departamento de Biblioteconomía, Documentación y Comunicación Audiovisual de la Universitat de Barcelona (UB) y colaborador docente de los Estudios de Información y Comunicación de la Universitat Oberta de Catalunya (UOC). Es subdirector de la revista El profesional de la información, y director de las colecciones de libros El profesional de la información y EPI Scholar (Editorial UOC). Autor de numerosos artículos y de varios libros. Es miembro del grupo de investigación consolidado Cultura y contenidos digitales de la Universitat de Barcelona.

http://orcid.org/0000-0002-8601-3990

Universitat de Barcelona, Facultat de Biblioteconomia i Documentació Melcior de Palau, 140. 08014 Barcelona, España jguallar@gmail.com

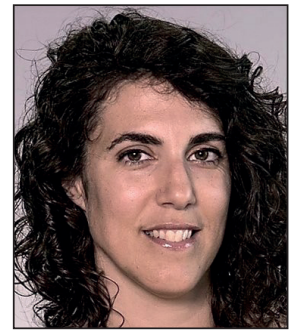

Núria Ferran-Ferrer es profesora en el Departamento de Ciencias de la Información y de la Comunicación de la Universitat Oberta de Catalunya (UOC) desde 2005. Obtuvo su doctorado europeo en 2010 en la Universitat de Barcelona con una etapa de investigación en la University of Sheffield. Participa en proyectos de investigación competitiva sobre ciencia abierta (Acceso abierto), ciencia ciudadana (Stem4Youth), participación política de los jóvenes en las redes sociales (Eurika) y uso de dispositivos móviles (ACT). Colabora con el Servei de Biblioteques de la Generalitat de Catalunya realizando estudios de usuarios.

http://orcid.org/0000-0002-9037-8837

Universitat Oberta de Catalunya Av. Tibidabo, 39-43. 08035 Barcelona, España nferranf@uoc.edu

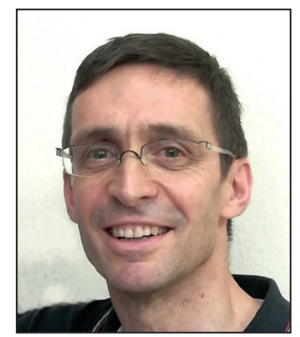

Ernest Abadal es catedrático de la Facultat de Biblioteconomia i Documentació de la Universitat de Barcelona, siendo actualmente decano de dicha Facultad. Licenciado en Filosofía, diplomado en Biblioteconomía y Documentación y doctor en Ciencias de la información. Es editor de la revista BiD y miembro del consejo asesor de diversas revistas (AIB Studi, Hipertext.net, El profesional de la información, entre otras). Dirige un proyecto de investigación sobre acceso abierto a la ciencia en España y es coordinador del grupo de investigación consolidado Cultura y contenidos digitales de la Universidad de Barcelona.

http://orcid.org/0000-0002-9151-6437

Universitat de Barcelona, Facultat de Biblioteconomia i Documentació Melcior de Palau, 140. 08014 Barcelona, España abadal@ub.edu

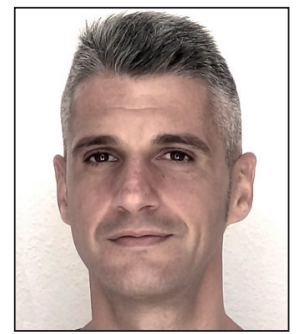

Adán Server es diplomado en Biblioteconomía y Documentación, licenciado en Documentación y postgrado en Tratamiento y gestión del patrimonio bibliográfico por la Universitat de Barcelona. Trabaja en la Biblioteca de l'Ateneu Barcelonès. Ha colaborado en proyectos de investigación impulsados por la Universitat de Barcelona y en la gestión editorial de revistas científicas. http://orcid.org/0000-0002-8476-9882 adanserver@gmail.com 


\title{
Resumen
}

Se presenta un estudio sobre la investigación en revistas de Información y Documentación en España. Se han analizado los artículos publicados entre 2012 y 2014 en las revistas Anales de documentación, BiD: textos universitaris de biblioteconomia i documentació, Cybermetrics, El profesional de la información (EPI), Revista española de documentación científica (REDC), Revista general de información y documentación (RGID) y Scire. Los principales temas tratados son: porcentaje de investigación, temáticas, aproximación metodológica cuantitativa o cualitativa, métodos de investigación y técnicas de investigación. Se muestran resultados para cada revista.

\section{Palabras clave}

Investigación; Información y Documentación; Revistas científicas; Temas de investigación; Métodos de investigación; Técnicas de investigación; España.

\begin{abstract}
A study about research in Library and Information Science in Spain is presented. Articles published between 2012 and 2014 have been analyzed in the journals Anales de Documentación, BiD: textos universitaris de biblioteconomia i documentació, Cybermetrics, El profesional de la información (EPI), Revista española de documentación científica (REDC), Revista general de información y documentación (RGID), and Scire. The main subjects studied are: percentage of original research, research topics, quantitative or quantitative methodological approach, research methods, and research techniques. Results are shown globally and from each journal.
\end{abstract}

\section{Keywords}

Research; Library and Information Science; Journals; Research topics; Research methods; Research techniques; Spain.

Guallar, Javier; Ferran-Ferrer, Núria; Abadal, Ernest; Server, Adán (2017). "Revistas científicas españolas de información y documentación: análisis temático y metodológico". El profesional de la información, v. 26, n. 5, pp. $947-960$.

https://doi.org/10.3145/epi.2017.sep.16

\section{Introducción y antecedentes}

Los estudios sobre análisis de métodos y técnicas de investigación utilizados en el área de información y documentación tienen una larga tradición. Järvelin, Vakkari y colaboradores (Järvelin; Vakkari, 1990; 1993; Toumaala; Järvelin; Vakkari, 2014) son seguramente los especialistas más destacados en este tema, ya que han llevado a cabo evaluaciones metodológicas de manera constante en su trayectoria académica, y sus categorizaciones se han tomado como referencia en buena parte de los estudios metodológicos realizados. Además de éstos, se ha publicado un gran número de trabajos similares sobre todo tipo de documentos (artículos, tesis, congresos, etc.), zonas geográficas y períodos cronológicos que ya fueron analizados de manera muy completa por Delgado-López-Cózar (2000a, 2000b, 2002) en unos estudios pioneros en España y actualizados en un artículo previo propio (Ferran-Ferrer et al., 2017).

Los estudios generales sobre revistas españolas en biblioteconomía y documentación han sido diversos. Giménez-Toledo y Román-Román (2000) evaluaron cinco revistas según dos modelos de análisis (Universidade de São Paulo y Colciencias) y establecieron un ranking. Delgado-López-Cózar (2001) llevó a cabo una descripción de las revistas y resaltó las deficiencias relativas al cumplimiento de las normas internacionales de publicación. Villagrá-Rubio y Sorli-Rojo (2003) analizaron la situación de las revistas en el período 1992-2001 respecto del grado de cumplimiento de indicadores de calidad editorial, del nivel de apertura de los comités editoriales y del reconocimiento de la revista entre la comunidad científica.
En lo que se refiere específicamente al análisis de métodos y técnicas de investigación en artículos de revista, Cano (1999) hizo un análisis de 354 artículos publicados en la Revista española de documentación científica (REDC) y en Documentación de las ciencias de la información en el período 1977-1994. Ríos-Hilario (2001) se volvió a centrar en la REDC en los años 1989-1999, y más tarde en el período 2000-2010 (Ríos-Hilario; Travieso-Rodríguez, 2013). GuaIlar (2003) aplicó la clasificación de métodos y técnicas de investigación de Abadal (2006) al análisis de artículos del campo de la documentación periodística publicados en revistas españolas.

Por otra parte, se pueden encontrar estudios específicos sobre artículos de revistas que analizan otras cuestiones como la autoría y los temas en la Revista española de documentación científica (Pérez-Álvarez-Ossorio, 1997; Jiménez-Hidalgo, 2007), en la Revista general de información y documentación (López-López et al., 2001), en BiD (Ollé-Castellà; Porras, 2008) y en Anales de documentación (González-Alcaide et al., 2008).

Sánchez-Vigil, Marcos-Recio y Olivera-Zaldua (2015) estudiaron la presencia del tema de comunicación y medios en revistas de documentación y Kawalec (2013) analizó los temas tratados en 1.051 artículos de revistas y también monografías del período 2000-10. Así mismo se puede hacer mención de otros textos sobre investigación en revistas, aunque no centrados en metodologías y/o en temáticas de investigación sino en otros aspectos, como el de Frías y Romero-Gómez (1998) que analiza las citas y las autorías de artículos en varias revistas del área. 
Las publicaciones anteriores se centran en artículos de revista, pero también se pueden encontrar análisis de la investigación en otros tipos de documentos: tesis doctorales (Román-Román; Sorli-Rojo, 1990); libros (Celestino-Angulo, 1995); proyectos y programas de investigación (Asensi-Artiga, 1997; Abellán-García; Serrano-Mascaraque; Ernica-Lafuente, 1997); resúmenes (Amat; Castillo-Blasco, 1997); actas de congresos (Cortés-Alonso, 1993; López-Gijón; Pérez-López; Ruiz-de-Villegas-del-Coso, 1995; Ríos-Hilario, 1998).

Forzosamente los estudios tienen que realizar una selección de títulos, y actualmente acostumbran a centrarse en las revistas de mayor calidad (indexadas en WoS o Scopus), como es nuestro caso.

En un trabajo previo (Ferran-Ferrer et al., 2017) se ofrece un análisis global y actualizado de las autorías, temas y metodologías de investigación en artículos publicados en las siete revistas españolas de mayor impacto del campo de información y documentación. En el texto que se presenta ahora queremos ofrecer un análisis pormenorizado de cada una de estas revistas para poder captar con mayor detalle su singularidad respecto de los temas, métodos y técnicas de investigación.

\section{Objetivos y metodología}

El objetivo general de este estudio es analizar las principales revistas españolas de información y documentación respecto a los temas, métodos y técnicas de investigación y relacionar los resultados con otros estudios similares.

Las preguntas de investigación formuladas son las siguientes:

- ¿Cuál es el porcentaje de textos de investigación publicados en las revistas españolas?
- ¿Cuáles son los temas que tienen mayor presencia en cada una de las revistas?

- ¿Qué aproximaciones metodológicas son las más habituales en cada una de las revistas?

- ¿Cuáles son los métodos de investigación más utilizados en cada una de las revistas?

- ¿Cuáles son las técnicas de investigación más utilizadas en cada una de las revistas?

El análisis se ha llevado a cabo en los artículos publicados en las revistas españolas de información y documentación indexadas en Social Sciences Citation Index (SSCI) de la Web of Science (WoS, de Clarivate Analytics) y en Scopus (de Elsevier). Las revistas analizadas son:

- Anales de documentación;

- BiD: textos universitaris de biblioteconomia i documentació;

- Cybermetrics;

- El profesional de la información (EPI);

- Revista española de documentación científica (REDC);

- Revista general de información y documentación (RGID);

- Scire.

Tan sólo dos, EPI y REDC, están incluidas tanto en Scopus como en $\mathrm{SSCl}$ de WoS. Se pueden encontrar los datos descriptivos de estas revistas en la tabla 1.

El período cubierto son tres años (2012-2014) y el total de artículos analizados es de 580. La distribución por revistas aparece recogida en la tabla 1.

Es significativa la presencia desigual de cada publicación en el total de la producción científica del campo de la información y la documentación española de mayor visibilidad internacional. Un alto número de artículos se publican en una sola revista, EPI (232 de 580, el 40\% del total) actualmente

Tabla 1. Descripción de las revistas españolas indexadas en SSCI (WoS) o Scopus con los artículos publicados entre 2012-2014 (\%)

\begin{tabular}{|c|c|c|c|c|c|c|}
\hline Revista & Abreviación & ISSN & Editor & Periodicidad & $\begin{array}{c}\text { N. de artículos } \\
(n=580)\end{array}$ & $\%$ artículos \\
\hline Anales de documentación & $A D$ & $\begin{array}{l}1575-2437 \\
1697-7904\end{array}$ & $\begin{array}{l}\text { Facultad de Comunicación y Docu- } \\
\text { mentación } \\
\text { Universidad de Murcia }\end{array}$ & Bianual & 48 & 8,3 \\
\hline $\begin{array}{l}\text { BiD: textos universitaris de } \\
\text { biblioteconomia i documen- } \\
\text { tació }\end{array}$ & $B I D$ & $\begin{array}{l}1575-5886 \\
0006-1778\end{array}$ & $\begin{array}{l}\text { Facultad de Biblioteconomía y Do- } \\
\text { cumentación de la Universitat de } \\
\text { Barcelona y Estudios de Ciencias de } \\
\text { la Información y Comunicación de } \\
\text { la Universitat Oberta de Catalunya }\end{array}$ & Bianual & 78 & 13,4 \\
\hline $\begin{array}{l}\text { Cybermetrics: International } \\
\text { journal of scientometrics, } \\
\text { informetrics and biblio- } \\
\text { metrics }\end{array}$ & Cyber & $1137-5019$ & $\begin{array}{l}\text { Instituto de Políticas y Bienes Públi- } \\
\text { cos (IPP) del Consejo Superior de } \\
\text { Investigaciones Científicas (CSIC) }\end{array}$ & Anual & 5 & 0,9 \\
\hline $\begin{array}{l}\text { El profesional de la infor- } \\
\text { mación }\end{array}$ & $E P I$ & $\begin{array}{l}1386-6710 \\
1699-2407\end{array}$ & $\begin{array}{l}\text { Ediciones Profesionales de la } \\
\text { Información SL }\end{array}$ & Bimestral & 232 & 40 \\
\hline $\begin{array}{l}\text { Revista española de docu- } \\
\text { mentación científica }\end{array}$ & $R E D C$ & $\begin{array}{l}0210-0614 \\
1988-4621\end{array}$ & $\begin{array}{l}\text { Consejo Superior de Investigacio- } \\
\text { nes Científicas (CSIC) }\end{array}$ & Trimestral & 111 & 19,1 \\
\hline $\begin{array}{l}\text { Revista general de informa- } \\
\text { ción y documentación }\end{array}$ & $R G I D$ & $\begin{array}{l}1132-1873 \\
1698-0921 \\
1988-2858\end{array}$ & $\begin{array}{l}\text { Facultad de Ciencias de la Do- } \\
\text { cumentación de la Universidad } \\
\text { Complutense de Madrid }\end{array}$ & Semestral & 50 & 8,6 \\
\hline $\begin{array}{l}\text { Scire: Representación y } \\
\text { organización del conoci- } \\
\text { miento }\end{array}$ & Scire & $1135-3716$ & $\begin{array}{l}\text { Facultad de Filosofía y Letras de } \\
\text { la Universidad de Zaragoza, Red } \\
\text { lbersid }\end{array}$ & Semestral & 56 & 9,7 \\
\hline
\end{tabular}


Tabla 2. Cuadro de clasificación: indicadores utilizados para el análisis de contenido de las revistas científicas de información y documentación

\begin{tabular}{|l|l|}
\hline \multicolumn{1}{|c|}{ Indicador } & \multicolumn{1}{c|}{ Valores del dominio } \\
\hline Investigación & Sí / No \\
\hline Tema & Ver Anexo 2 \\
\hline Método de investigación - en resumen & Sí / No \\
\hline Método de investigación - Aproximación & Cualitativa / Cuantitativa \\
\hline Método de investigación - Tipo & Ver Anexo 3 \\
\hline Técnica de investigación - Tipo & Ver Anexo 4 \\
\hline
\end{tabular}

con una periodicidad de 6 números al año. Le sigue, con 111 artículos y periodicidad trimestral REDC (casi el $20 \%$ de la producción total). Sumando ambas se alcanza el $60 \%$ de los artículos de la muestra estudiada.

El resto mantienen proporciones más parecidas entre sí, publicando entre 48 y 78 artículos en el período analizado, con la notable excepción de Cyber, de periodicidad anual, que sólo publica 5 artículos en este período (no llega al 1\% de la producción global).

Para cada uno de los 580 artículos se ha realizado un análisis de contenido a partir de los indicadores que se muestran en la tabla 2.

En primer lugar se analizó si los artículos podían considerarse de investigación o no (indicador "investigación") a partir de si la metodología era explícita o implícita en el cuerpo del artículo y/o si aportaba una contribución al conocimiento, siguiendo la definición operativa propuesta por Delgado-López-Cózar (2002) basándose en Peritz (1981). Se analizó también la presencia de la metodología en el resumen, si estaba presente en el mismo o podía deducirse, o si, por el contrario, la explicación del método estaba totalmente ausente (indicador: método de investigación-resumen).

En segundo lugar se codificaron todos los artículos en función de su tema partiendo del Tesauro de biblioteconomía y documentación (Mochón-Bezares; Sorli-Rojo, 2002). Este lenguaje controlado se creó para realizar el análisis de los documentos de la base de datos sobre biblioteconomía y documentación ISOC-DC producida por el Cindoc (CSIC) desde 1975 y cubre la ausencia de léxicos documentales en español que abarquen todos los campos semánticos representados en los textos científico-técnicos publicados en este país, muchos de los cuales están recogidos en la mencionada base de datos. De hecho una buena parte de los textos analizados en este artículo ya estaban indexados temáticamente en la base de datos.

A continuación se analizó la aproximación metodológica, los métodos y las técnicas de investigación utilizados en cada artículo. Estos tres indicadores parten del análisis de las metodologías de la investigación en ciencias de la información y la documentación (library and information sciences, LIS). Se han considerado manuales de metodología de la investigación (Busha; Harter, 1990; Powell, 1997), estudios sobre la aplicación de las metodologías en publicaciones (Feehan et al., 1987; Järvelin; Vakkari, 1990; Blake, 1994; Dimitroff, 1995; Hider; Pymm, 2008; etc.) y estudios de revisión metodológica (Delgado-López-Cózar (2000b).
Tabla 3. Resultados del test de fiabilidad

\begin{tabular}{|c|c|}
\hline \multicolumn{2}{|c|}{ Reliability test } \\
\hline Indicador & $\%$ de coincidencias \\
\hline Investigación (sí/no) & 100 \\
\hline Aproximación metodológica & 100 \\
\hline Método de investigación & 80 \\
\hline Técnicas de investigación & 93 \\
\hline
\end{tabular}

En la codificación de los contenidos participaron dos codificadores. Se realizó un test de fiabilidad (reliability) (Krippendorff, 2004) en todos los artículos de un número de revista escogido al azar (tabla 3): el número 28 de la revista BiD con 15 artículos. El test para comprobar similitudes y diferencias situó las coincidencias de 3 indicadores evaluados por encima del $90 \%$, siendo aceptables todos los valores de estos indicadores. Sólo el indicador del método de investigación quedó por debajo de este coeficiente, en el $80 \%$, resultado que hace aceptables los valores para la mayoría de las codificaciones realizadas por los dos investigadores (Neuendorf, 2002).

\section{Resultados y discusión}

\subsection{Proporción de artículos de investigación}

Las revistas analizadas presentan en conjunto un porcentaje aproximado de $68 \%$ de artículos de investigación (tabla 4). Este dato señala una importante presencia de la investigación en las principales revistas españolas de información y documentación, superior a los porcentajes de la mayoría de estudios en el ámbito español realizados en fechas que tienen más de 10 años de distancia, como Ríos-Hilario (2001) en que los artículos de investigación no llegaban a la mitad (45,5\%), Delgado-López-Cózar en 2002 (44,6\%), el mismo autor en 2003 (39\%) y Guallar en 2003 (34,7\%). La década larga transcurrida entre estos datos y los que aquí se ofrecen, aun con la disparidad de muestras de análisis de cada estudio, parece indicar que ha habido un importante crecimiento de la actividad de investigación en la bibliografía especializada de información y documentación en España en estos años. Esta tendencia también se pone de manifiesto en el estudio de Ríos-Hilario y Travieso-Rodríguez (2013) para una sola revista (REDC), que alcanza un $81,9 \%$. Si comparamos nuestros datos con el entorno internacional, vemos que el porcentaje de investigación está tan sólo 4 puntos por debajo del valor (72\%) que consta en el estudio de Toumalaa, Järvelin y Vakkari (2014) a partir de datos de 2005.

En números absolutos, en los tres años estudiados se publicaron 394 artículos de investigación de alta visibilidad internacional en el área. La revista que publicó mayor número de trabajos de investigación fue EPI con 145, una cifra bastante notable que representa algo menos del $40 \%$ del total de los artículos de investigación publicados en España (un $36,8 \%$ ). También tiene una cifra bastante elevada la $R E D C$ con 102 artículos, lo que representa una cuarta parte del total $(25,9 \%)$. El resto de las revistas publica en el período estudiado un número de trabajos de investigación que os- 
Tabla 4. Artículos de investigación en el global de las revistas científicas españolas 2012-2014. Por orden de producción.

\begin{tabular}{|l|c|c|}
\hline \multicolumn{1}{|c|}{ Revista } & $\begin{array}{c}\text { Artículos } \\
\text { investigación } \\
\text { (n=394) }\end{array}$ & $\begin{array}{c}\text { Artículos } \\
\text { no-investigación \% } \\
\text { (n=186) }\end{array}$ \\
\hline EPI & 36,8 & 46,8 \\
\hline REDC & 25,9 & 4,8 \\
\hline Scire & 9,9 & 9,1 \\
\hline BiD & 9,4 & 22,0 \\
\hline RGID & 9,1 & 7,5 \\
\hline AD & 7,6 & 9,7 \\
\hline Cyber & 1,3 & 0,0 \\
\hline Total & 68,0 & 32,0 \\
\hline
\end{tabular}

cila entre 30 y 39, exceptuando Cyber que queda en último lugar con sus 5 artículos.

$E P I$ también es la revista que publica más trabajos de no investigación: 87 , acercándose su porcentaje casi al $50 \%$ del total (un 46,8\%). Por su parte, los 41 artículos de no investigación de BiD representan el $22 \%$ de los trabajos de esta categoría, y el resto de las revistas no llegan a porcentajes del $10 \%$ del total.

En el porcentaje de publicación de investigación dentro de la propia revista (tabla 5), Cyber invierte la última posición que tenía en cuanto a número de artículos, y pasa a ocupar el primer lugar al publicar un $100 \%$ de trabajos que son fruto de investigación. También con un alto porcentaje, cercano al $90 \%$ y muy meritorio teniendo en cuenta su alta producción, se encuentra la REDC $(91,9 \%)$, mientras que en niveles también altos, pero entre el $62 \%$ y el $72 \%$, se encuentra el resto de las publicaciones, con la excepción de $B i D$, que con un $47,4 \%$ de artículos de investigación, es la única revista que (por poco) publica más trabajos que no son de investigación que los que sí lo son.

\subsection{Tema}

La temática con mayor proporción de artículos de investigación son los estudios métricos (19\%), siendo las publicaciones con más artículos Cyber, con el $100 \%$ de artículos centrados en este tema y $\operatorname{REDC}(33,3 \%)$. Así mismo, todas las publicaciones analizadas tienen artículos sobre métricas, con un intervalo entre $7,7 \%$ y $33,3 \%$ (tabla 6). Este tema según señalaba Delgado-López-Cózar (2002) ha distinguido la investigación española internacionalmente.

\section{La temática con mayor proporción de ar- tículos de investigación son los estudios métricos}

Las fuentes de información, que es el segundo tema más abundante en producción científica global (14,4\%), ocupa el primer lugar como más habitual en las revistas $A D(36,7 \%)$, BiD $(25 \%)$ y RGID $(23,7 \%)$. Éste es también el tema más extendido en el estudio de Kawalec (2013), llegando a un 24\%.
Tabla 5. Artículos de investigación y no-investigación en cada revista \% $(n=580)$. Por orden de porcentaje de investigación

\begin{tabular}{|l|c|c|}
\hline \multicolumn{1}{|c|}{ Revista } & Investigación \% & No-investigación \% \\
\hline Cyber $(\mathrm{n}=5)$ & 100,0 & 0,0 \\
\hline REDC $(\mathrm{n}=111)$ & 91,9 & 8,1 \\
\hline RGID $(\mathrm{n}=50)$ & 72,0 & 28,0 \\
\hline Scire $(\mathrm{n}=56)$ & 69,6 & 30,4 \\
\hline$A D(\mathrm{n}=48)$ & 62,5 & 37,5 \\
\hline$E P I(\mathrm{n}=232)$ & 62,5 & 37,5 \\
\hline$B i D(\mathrm{n}=78)$ & 47,4 & 52,6 \\
\hline
\end{tabular}

Esta autora no distingue si los artículos son de investigación o no, y por tanto las cifras de su estudio y el presente son difíciles de comparar, aunque es significativo que ambos estudios sitúen las fuentes de información como una de las materias más habituales en la investigación en España.

Si comparamos con los temas más recurrentes en el estudio de Cano (1999), que eran la recuperación de la información, la descripción de servicios y los estudios sobre la comunicación científica, ha cambiado de prioridad y estos temas dejan de estar en las primeras posiciones.

Nuestro estudio coincide con Ollé-Castellà y Porras (2008) en la importancia de las temáticas relacionadas con las tecnologías de la información, sin embargo la diferencia es clara en relación con los estudios métricos que en Ollé-Castellà y Porras aparece como poco habitual.

La materia más recurrente en los artículos de no investigación son las tecnologías de la información y la comunicación $(18,8 \%)$ donde habitualmente se explican o describen productos y servicios. Las revistas con más artículos de no investigación publicados con este tema son Scire $(29,4 \%)$, $\operatorname{REDC}(22,2 \%), \operatorname{EPI}(20,7 \%)$ y $\operatorname{BiD}(18 \%)$.

\subsection{Análisis metodológico}

\subsubsection{Presencia de la metodología en el resumen}

Las revistas con más presencia de la metodología en el resumen (tabla 7), son: Cyber $(100 \%)$, BiD $(91,9 \%)$ y $R E D C$ $(81,4 \%)$. Y las que tienen más artículos que no hacen referencia a la metodología en el mismo, en proporciones que se acercan al $50 \%$, son Scire $(48,7 \%)$ y $R G I D(44,4 \%)$. En conjunto, casi el $70 \%$ de los artículos publicados en las publicaciones estudiadas tratan la metodología en el resumen.

Se puede considerar a nivel general que existe una relación entre la existencia de investigación en un artículo y la presencia de algún tipo de indicación metodológica en el resumen del mismo.

Otra cuestión a comentar en este apartado es que la existencia de resúmenes estructurados en los que la revista indica a los autores que incluyan un apartado de explicaciones metodológicas, como sucede en BiD, no garantiza una alta proporción de investigación en la revista $(47,4 \%$ en $B i D$, tabla 5). 
Tabla 6. Temas de investigación por revista $(\%)(n=580)$

\begin{tabular}{|c|c|c|c|c|c|c|c|c|c|c|c|c|c|c|c|c|}
\hline & & D & & iD & $E F$ & PI & REL & $D C$ & $R C$ & DID & Sc & ire & & ber & To & tal \\
\hline & $\begin{array}{c}\text { Inv } \\
(n=30)\end{array}$ & $\begin{array}{l}\text { No-inv } \\
(n=18)\end{array}$ & $\begin{array}{c}\text { Inv } \\
(n=36)\end{array}$ & $\begin{array}{l}\text { No-inv } \\
(n=41)\end{array}$ & $\begin{array}{c}\text { Inv } \\
(n=145)\end{array}$ & $\begin{array}{l}\text { No-inv } \\
(n=87)\end{array}$ & $\begin{array}{c}\text { Inv } \\
(n=102)\end{array}$ & $\begin{array}{c}\text { No-inv } \\
(n=9)\end{array}$ & $\begin{array}{c}\text { Inv } \\
(n=38)\end{array}$ & $\begin{array}{l}\text { No-inv } \\
(n=12)\end{array}$ & $\begin{array}{c}\text { Inv } \\
(n=39)\end{array}$ & $\begin{array}{l}\text { No-inv } \\
(n=17)\end{array}$ & $\begin{array}{l}\text { Inv } \\
(n=5)\end{array}$ & $\begin{array}{c}\text { No-inv } \\
(n=0)\end{array}$ & $\begin{array}{c}\text { Inv } \\
(n=394)\end{array}$ & $\begin{array}{c}\text { No-inv } \\
(n=186)\end{array}$ \\
\hline $\begin{array}{l}\text { Fuentes de } \\
\text { información y } \\
\text { recursos }\end{array}$ & 36,7 & 22,2 & 25,0 & 12 & 12,4 & 13,8 & 6,9 & 0,0 & 23,7 & 16,7 & 5,1 & 23,5 & 0 & 0 & 14,43 & 15,59 \\
\hline $\begin{array}{l}\text { Estudios } \\
\text { métricos }\end{array}$ & 10,0 & 0,0 & 13,9 & 4 & 13,1 & 4,6 & 33,3 & 0,0 & 13,2 & 0,0 & 7,7 & 0,0 & 100 & 0 & 18,99 & 2,15 \\
\hline $\begin{array}{l}\text { Tecnologías } \\
\text { de la } \\
\text { información }\end{array}$ & 3,3 & 0,0 & 5,6 & 18 & 15,9 & 20,7 & 5,9 & 22,2 & 7,9 & 8,3 & 5,1 & 29,4 & 0 & 0 & 9,11 & 18,82 \\
\hline $\begin{array}{l}\text { Técnicas auxi- } \\
\text { liares y otras } \\
\text { disciplinas }\end{array}$ & 13,3 & 11,1 & 8,3 & 9 & 8,3 & 10,3 & 22,5 & 22,2 & 10,5 & 25,0 & 5,1 & 11,8 & 0 & 0 & 11,90 & 10,75 \\
\hline $\begin{array}{l}\text { Comunica- } \\
\text { ción }\end{array}$ & 3,3 & 11,1 & 5,6 & 10 & 21,4 & 11,5 & 2,9 & 0,0 & 2,6 & 8,3 & 2,6 & 0,0 & 0 & 0 & 10,13 & 7,53 \\
\hline $\begin{array}{l}\text { Fundamen- } \\
\text { tos teóricos } \\
\text { y aspectos } \\
\text { generales }\end{array}$ & 3,3 & 0,0 & 0,0 & 2 & 6,2 & 2,3 & 4,9 & 11,1 & 7,9 & 8,3 & 48,7 & 17,6 & 0 & 0 & 9,62 & 3,76 \\
\hline $\begin{array}{l}\text { Unidades de } \\
\text { información y } \\
\text { servicios }\end{array}$ & 10,0 & 27,8 & 8,3 & 15 & 9,7 & 17,2 & 2,0 & 0,0 & 2,6 & 16,7 & 2,6 & 0,0 & 0 & 0 & 3,04 & 14,52 \\
\hline Profesionales & 3,3 & 11,1 & 16,7 & 5 & 5,5 & 5,7 & 3,9 & 11,1 & 10,5 & 8,3 & 2,6 & 11,8 & 0 & 0 & 6,08 & 7,53 \\
\hline $\begin{array}{l}\text { Acceso y recu- } \\
\text { peración de la } \\
\text { información }\end{array}$ & 3,3 & 5,6 & 0,0 & 7 & 6,2 & 8,0 & 4,9 & 22,2 & 2,6 & 0,0 & 5,1 & 5,9 & 0 & 0 & 4,30 & 7,53 \\
\hline $\begin{array}{l}\text { Proceso } \\
\text { técnico }\end{array}$ & 0,0 & 0,0 & 8,3 & 2 & 0,0 & 2,3 & 4,9 & 0,0 & 5,3 & 0,0 & 15,4 & 0,0 & 0 & 0 & 4,05 & 7,53 \\
\hline Archivística & 0,0 & 11,1 & 5,6 & 2 & 0,7 & 2,3 & 1,0 & 0,0 & 13,2 & 8,3 & 0,0 & 0,0 & 0 & 0 & 6,33 & 1,61 \\
\hline Usuarios & 13,3 & 0,0 & 2,8 & 1 & 0,7 & 1,1 & 6,9 & 11,1 & 0,0 & 0,0 & 0,0 & 0,0 & 0 & 0 & 2,03 & 2,69 \\
\hline
\end{tabular}

\subsubsection{Aproximación metodológica}

Teniendo en cuenta sólo los trabajos que se han considerado de investigación (394 artículos de los 580 analizados), en un estudio anterior (Ferran-Ferrer et al., 2017) señalamos que se constata un alto equilibro entre los paradigmas cuantitativo y cualitativo en la investigación en documentación ( $45 \%$ vs. $44 \%$ ) y que sólo un $10 \%$ de los trabajos se han realizado mediante la combinación de ambos paradigmas.

La investigación descriptiva, que en conjunto es la más empleada, es el método más utilizado en $B i D, A D$ y $E P I$, y mantiene proporciones destacables en el resto

Mirando las revistas individualmente (tabla 8), se observa que en Cyber la totalidad de su producción es de investigación cuantitativa y en 3 revistas más es la investigación mayoritaria: $\operatorname{REDC}(60,8 \%), A D(56,7 \%)$ y $\operatorname{BiD}(51,4 \%)$. Por el contrario, la proporción de aproximación cualitativa por encima de la cuantitativa se da en las 3 revistas restantes: dos de ellas en proporciones muy altas: $R G I D(63,9 \%)$ y Scire $(61,5 \%)$, y la tercera, $E P I$ en un $49,7 \%$.

Por último, las revistas con mayor proporción de artículos realizados combinando ambas aproximaciones son $R G I D$, EPI y BiD, que sobrepasan el $10 \%$ de su producción.

Una mayoría de las publicaciones españolas del área recogen adecuadamente la pluralidad de enfoques posibles de investigación existentes en documentación

\subsubsection{Métodos de investigación por revistas}

La tabla 9 muestra la distribución de métodos de investigación en cada revista teniendo en cuenta sólo los artículos de investigación. Aquí se aprecian diferencias significativas dependiendo de las publicaciones en la utilización de unos métodos u otros. 
Si observamos en primer lugar los métodos más utilizados en cada publicación, vemos que la investigación descriptiva, que en conjunto es la más empleada $(40,1 \%$ del total de artículos), aparece como el método más empleado en tres revistas: $B i D$ (59\% de sus artículos), $A D(53 \%)$ y $E P I$ (47\%), y mantiene proporciones destacables en el resto. En dos revistas el método más empleado es la bibliometría y cibermetría (Cyber, 60\% y REDC, 35,64\%); mientras que la investigación histórica es el método más utilizado en $R G I D$ (36\%) y la investigación teórica o conceptual en Scire (32\%).

Por otra parte, si observamos la presencia de los diversos métodos dentro de cada publicación, vemos que sólo una de ellas tiene representación de todos $(E P I)$, aunque en tres revistas más hay representación de todos menos uno (BiD, REDC y Scire) y en otra más de todos menos 2 (RGID). En este sentido, se puede considerar que una mayoría de las publicaciones españolas del área recogen adecuadamente la pluralidad de enfoques posibles de investigación existentes en documentación. Sólo quedan fuera de esta diversidad dos revistas: $A D$, donde faltaría representación de cuatro métodos, y muy especialmente, Cyber, que sólo ha publicado artículos de dos métodos (bibliometría y descriptiva), sin ninguna presencia del resto.

En Cyber y REDC el método de investigación más empleado es la bibliometría y cibermetría, en $R G I D$ la investigación histórica y en Scire la investigación teórica o conceptual

Estos datos sugieren dos tendencias:

- cierta preferencia de las publicaciones por algunos métodos concretos de investigación, que aparecen priorizados entre el conjunto de su producción;

- dos comportamientos diferentes respecto a la presencia de la diversidad de enfoques posibles de investigación: una mayoría de revistas publica investigaciones con una alta diversidad de métodos y otras son más restrictivas o especializadas.

Esta investigación coincide con las observaciones de otros investigadores, como Cano (1999) que señala la baja pre-

Tabla 7. Método de investigación mencionado en el resumen de los artículos de investigación por cada una de las revistas $(n=394)(\%)$

\begin{tabular}{|l|c|c|}
\hline \multicolumn{1}{|c|}{ Revistas } & Sí en resumen & No en resumen \\
\hline$A D$ & 70,0 & 30,0 \\
\hline BiD & 91,9 & 8,1 \\
\hline Cyber & 100,0 & 0,0 \\
\hline EPI & 62,1 & 37,9 \\
\hline REDC & 81,4 & 18,6 \\
\hline RGID & 55,6 & 44,4 \\
\hline Scire & 51,3 & 48,7 \\
\hline Total & 69,3 & 30,7 \\
\hline
\end{tabular}

sencia de trabajos experimentales (5\%) y la alta presencia de estudios descriptivos (40\%) y que también se puso de manifiesto en el estudio de Delgado-López-Cózar (2000b).

\section{El análisis de contenido es la técnica de investigación más empleada globalmen- te en el conjunto de revistas}

\subsubsection{Técnicas de investigación por revistas}

Se aprecian algunas diferencias llamativas entre revistas en lo que se refiere a técnicas de investigación (tabla 10). El análisis de contenido es la técnica más empleada globalmente considerando el conjunto de revistas estudiadas (23,6\%) y es asimismo la más utilizada en los artículos publicados en $A D$ y $R G I D$ (hasta el $50 \%$ de sus artículos en ambas), así como en BiD (32\%) y en EPI (26\%). Tienen porcentajes altos de uso a nivel global también la interpretación de textos y el análisis de citas o enlaces (19,1 y 17,2\% respectivamente). Por revistas, estas técnicas son las más empleadas en varias revistas: el análisis de citas o enlaces es la más utilizada en Cyber (80\%) y en REDC (35\%), y en Scire la interpretación de textos (36\%).

Otro dato de interés es que la única revista que tiene representación de todas las técnicas de investigación es EPI y la revista con menos representación de técnicas es Cyber, que se concentra en el análisis de citas o enlaces (80\%). En conjunto, la distribución de técnicas por revistas confirma y complementa el análisis que hemos visto antes de los métodos.

Es remarcable asimismo que la media de técnicas utilizadas por artículo es de 1,2, siendo pues poco habitual la utilización de más de una técnica por artículo.

\subsection{Perfil temático y metodológico de cada revista}

En este apartado se muestra una aproximación a los rasgos más distintivos de cada revista en relación con los elementos estudiados:

\section{Anales de documentación (AD)}

Ha publicado 48 artículos en el período analizado, representando el $7,6 \%$ de la producción global de investigación

Tabla 8. Orientación de los métodos de investigación por cada revista (\%) $(n=394)$

\begin{tabular}{|l|c|c|c|}
\hline & Cuantitativa & Cualitativa & Combinación \\
\hline$A D$ & 56,7 & 36,7 & 6,7 \\
\hline BiD & 51,4 & 37,8 & 10,8 \\
\hline Cyber & 100,0 & 0,0 & 0,0 \\
\hline EPI & 38,6 & 49,7 & 11,7 \\
\hline REDC & 60,8 & 31,4 & 7,8 \\
\hline RGID & 22,2 & 63,9 & 13,9 \\
\hline Scire & 33,3 & 61,5 & 5,1 \\
\hline
\end{tabular}


Tabla 9. Métodos de investigación por revistas ( $n=394) \%$

\begin{tabular}{|c|c|c|c|c|c|c|c|c|}
\hline Método de investigación & $A D$ & BiD & Cyber & EPI & REDC & RGID & Scire & Total \\
\hline Descriptiva & 53,33 & 59,46 & 40,00 & 47,26 & 30,69 & 27,78 & 17,50 & 40,10 \\
\hline Bibliometría y cibermetría & 13,79 & 13,51 & 60,00 & 11,64 & 35,64 & 13,89 & 15,00 & 19,00 \\
\hline Teórica o conceptual & 0,00 & 5,41 & 0,00 & 10,96 & 13,86 & 2,78 & 32,50 & 11,70 \\
\hline Evaluativa & 0,0 & 5,41 & 0,00 & 8,90 & 13,86 & 8,33 & 7,50 & 8,90 \\
\hline Diseño de sistemas de información & 6,90 & 5,41 & 0,00 & 12,33 & 2,97 & 2,78 & 17,50 & 8,40 \\
\hline Histórica & 13,79 & 5,41 & 0,00 & 0,00 & 0,99 & 36,11 & 0,00 & 5,10 \\
\hline Revisión de la literatura & 13,79 & 2,70 & 0,00 & 5,48 & 2,97 & 2,78 & 5,00 & 4,80 \\
\hline Bibliográfica & 0,00 & 0,00 & 0,00 & 0,68 & 5,56 & 0,00 & 2,5 & 1,00 \\
\hline Experimental & 0,00 & 2,70 & 0,00 & 2,05 & 0,00 & 0,00 & 0,00 & 1,00 \\
\hline
\end{tabular}

y un $9,7 \%$ de la no investigación. Su temática más habitual son las fuentes de información (58,9\%), seguida por unidades de información $(37,8)$ y las técnicas auxiliares y otras disciplinas $(24,4 \%)$. Son datos comparables sólo en parte con los del estudio de González-Alcaide et al. (2008), ya que sigue categorías diferentes a las nuestras (por ejemplo, divide fuentes de información por tipos) y en el que se señalaba como principal tema las tecnologías de la información.

\section{Anales es una revista decantada hacia las investigaciones descriptivas y el aná- lisis de contenido}

$A D$ destaca por ser la revista que presenta menos variedad de métodos y de técnicas de entre todas las publicaciones analizadas, sólo después de Cyber (que recordemos, es un caso especial, por su bajísima producción). Los métodos más utilizados son la investigación descriptiva con un porcentaje altísimo (53,3\%), seguida de la bibliometría y cibermetría, la investigación histórica y la revisión de la bibliografía.
En cuanto a técnicas, tiene una alta presencia el análisis del contenido (50\% de sus artículos), y le siguen la interpretación de textos, análisis de citas o enlaces y la encuesta.

Se caracterizaría por tanto por ser una revista decantada hacia las investigaciones descriptivas y el análisis de contenido, con poca variedad de metodologías en el conjunto de su publicación.

\section{BiD: textos universitaris de biblioteconomia i documenta- ció (BiD)}

Se analizaron 78 artículos (13,4\% de la producción total). De ellos, 37 son artículos de investigación $(47,4 \%)$ y 41 de no investigación $(52,6 \%)$, siendo la única revista con una proporción ligeramente superior de no-investigación. Se caracteriza también por ser la única publicación que requiere que los autores presenten un resumen estructurado en secciones (objetivos, metodología, resultados). Entre los artículos que se han considerado de investigación, el tema más presente es fuentes de información (25\%), mientras que entre los artículos de no-investigación los temas preferidos son tecnologías de la información (18\%) y unidades de información y servicios (15\%), categorías temáticas que coinciden con las obtenidas para esta revista en el estudio de Ollé-Castellà y Porras (2008).

Tabla 10. Técnicas de investigación por revistas ( $n=487$ técnicas) (total artículos=394) (\%)

\begin{tabular}{|c|c|c|c|c|c|c|c|c|}
\hline Técnicas de investigación & $A D$ & BiD & Cyber & EPI & REDC & RGID & Scire & Total \\
\hline Análisis de contenido & 50,0 & 32,0 & 0,0 & 26,0 & 23,0 & 50,0 & 23,0 & 23,6 \\
\hline Interpretación de textos & 20,0 & 22,0 & 0,0 & 22,0 & 20,0 & 36,0 & 36,0 & 19,1 \\
\hline Análisis de citas & 13,0 & 14,0 & 80,0 & 16,0 & 35,0 & 14,0 & 18,0 & 17,2 \\
\hline Encuesta & 17,0 & 16,0 & 0,0 & 17,0 & 17,0 & 5,6 & 7,7 & 11,9 \\
\hline Análisis y diseño de sistemas de información & 7,0 & 8,1 & 0,0 & 12,0 & 5,9 & 8,3 & 18,0 & 8,0 \\
\hline Estudio de caso & 3,0 & 14,0 & 20,0 & 14,0 & 8,8 & 5,6 & 5,1 & 7,8 \\
\hline Entrevista & 0,0 & 5,4 & 0,0 & 10,0 & 4,9 & 2,8 & 5,1 & 5,0 \\
\hline Análisis de logs & 0,0 & 2,7 & 20,0 & 4,0 & 2,0 & 2,8 & 0,0 & 2,3 \\
\hline Observación & 0,0 & 2,7 & 0,0 & 1,0 & 1,0 & 2,8 & 2,6 & 1,3 \\
\hline Análisis secundario & 0,0 & 0,0 & 0,0 & 1,0 & 2,0 & 2,8 & 0,0 & 1,0 \\
\hline Experimento & 0,0 & 2,7 & 0,0 & 3,0 & 0,0 & 0,0 & 0,0 & 1,0 \\
\hline Grupo de discusión & 0,0 & 2,7 & 0,0 & 1,0 & 0,0 & 0,0 & 2,6 & 0,9 \\
\hline Estudio Delphi & 0,0 & 0,0 & 0,0 & 1,0 & 1,0 & 0,0 & 0,0 & 0,6 \\
\hline
\end{tabular}

La asignación de las categorías es múltiple (es decir, un artículo puede emplear varias técnicas de investigación), por lo que los porcentajes suman más de 100 
En los artículos de investigación, predomina la investigación cuantitativa y se caracteriza especialmente por una gran diversidad de métodos ( 8 métodos de 9 ), siendo el mayoritario el descriptivo $(59,5 \%)$ y muy por detrás el bibliométrico $(13,5 \%)$. Sobre las técnicas utilizadas, BiD muestra una gran variedad de ellas para recoger o analizar datos, siendo las más empleadas: análisis de contenido (32\%), interpretación de textos (22\%) y encuestas (16\%).

\section{BiD tiene alta variedad en sus artículos, tanto en investigación y no investigación como en temáticas, métodos y técnicas}

Se caracteriza por ser una revista con alta variedad en sus artículos, tanto en investigación y no investigación como en las temáticas, métodos y técnicas empleados en sus artículos.

\section{Cybermetrics: International journal of scientometrics, in- formetrics and bibliometrics (Cyber)}

Revista de la que llama la atención su escasa producción: tan sólo 5 artículos en el período 2012-2014, que no llega al $1 \%$ de la producción global estudiada. Todos sus artículos pueden considerarse científicos, y en sus resúmenes aparece explícita la metodología. El tema dominante son los estudios métricos.

\section{Cybermetrics presenta un perfil muy es- pecializado y claramente orientado a la investigación en estudios métricos}

En clara relación con ello, predomina como método la investigación en bibliometría y cibermetría, mientras que las técnicas se concentran en análisis de citas (80\%), junto a análisis de logs (20\%) y estudios de caso (20\%).

Se trata por tanto de una publicación con un perfil muy especializado, selectivo y claramente orientado a la investigación en estudios métricos.

\section{El profesional de la información (EPI)}

Al publicar 6 números al año, es la revista con mayor producción científica de las estudiadas. En los 3 años analizados en este estudio, sus 232 artículos representan casi el $40 \%$ del total. El peso cuantitativo de EPI en el conjunto de la producción del área es por tanto evidente. La proporción de artículos de investigación es del $62,5 \%$ por un $37,5 \%$ de artículos de no investigación mientras que la proporción entre investigación cuantitativa y cualitativa se decanta a favor de esta última $(49,7 \%$ frente a $38,6 \%$, más un $11,7 \%$ de artículos de combinación de ambas).

En temas, EPI se diferencia claramente del resto por la alta presencia de artículos de comunicación (21,4\% de investigación y $11,5 \%$ de no investigación), lo que le confiere una singularidad temática dentro del sector. No obstante, la diversidad de temas considerados está igualmente representada en la revista, destacando también tecnologías de la información.
EPI sobresale especialmente por presentar una gran variedad de métodos y de técnicas de investigación en sus artículos. De hecho es la única publicación analizada con representación de todos los métodos y de todas las técnicas. Entre ellos, el método mayoritario es la investigación descriptiva $(47,26 \%)$ y la técnica más utilizada el análisis de contenido (26\%). Les siguen otros métodos, como diseño de sistemas de información, bibliometría y cibermetría, e investigación teórica, y otras técnicas como interpretación de textos, encuesta, análisis de citas o enlaces y estudio de caso. Es casi la única revista de la muestra donde se pueden ver ejemplos de los métodos y técnicas más minoritarios como son la investigación experimental, el grupo de discusión, o el estudio Delphi.

\section{$E P I$ es la única publicación analizada con representación de todos los métodos y de todas las técnicas de investigación}

En suma, EPI se caracterizaría no sólo por su peso cuantitativo en el área sino por ser la revista con una mayor variedad temática y de métodos y de técnicas de investigación.

\section{Revista española de documentación científica (REDC)}

Se han analizado 111 artículos de esta revista, casi el 20\% de la producción total (la segunda del área después de EPI) y de ellos 102 (91,9\%) pueden considerarse artículos de investigación, un dato relevante dada su alta producción. Los datos confirman la tendencia hacia el progresivo aumento del porcentaje de artículos de investigación en la revista que se aprecia claramente en los estudios de Ríos-Hilario (2001), que situaba este porcentaje en algo menos de la mitad, el 45,5\%, para las publicaciones del período 1989-1999, y Ríos-Hilario y Travieso-Rodríguez (2013), que señalan ya un 81,9\% para el período 2000-2010.

REDC destaca por ser una revista con fuerte proporción de la investigación cuantitativa $(60,8 \%$, además de otro $7,8 \%$ en combinación con cualitativa), sólo por detrás de Cybermetrics. Esta presencia del paradigma cuantitativo era muy superior en el primer estudio de Ríos-Hilario sobre la revista para los años 90 del siglo pasado: un 96,6\% (Ríos-Hilario, 2001) y se va aproximando a nuestros datos en una ligera tendencia a la baja, en su estudio realizado una década después: 82,4\% para el período 2000-2010 (Ríos-Hilario; Travieso-Rodríguez, 2013).

\section{REDC se caracteriza por una fuerte pre- sencia de los estudios métricos en temá- tica, métodos, técnicas y aproximación metodológica cuantitativa}

En relación con lo anterior, se constata una importante presencia de los estudios métricos tanto en cuanto a temática $(33,3 \%)$ como en cuanto a métodos (bibliometría y cibermetría, 35,6\%) y técnicas de investigación (análisis de citas o enlaces, $35 \%$ ). La diferente categorización de temas y metodología en los estudios de Cano y de Ríos-Hilario no permite 
una clara comparación, si bien, respecto a la primera autora, su categoría temática de comunicación científica $(23,28 \%)$, se puede interpretar como cercana a la nuestra de estudios métricos (33,3\%) (Cano, 1999); y respecto a la segunda autora cabe destacar que señala que "muchas de las investigaciones son trabajos bibliométricos" (Ríos-Hilario, 2001).

Otros métodos presentes en la revista en proporciones destacadas son la investigación descriptiva, la teórica y la evaluativa; y en cuanto a técnicas, el análisis de contenido, la interpretación de textos y la encuesta.

REDC se caracterizaría por una fuerte presencia de todo lo que representan los estudios métricos (en temática, métodos, técnicas y aproximación metodológica cuantitativa). Esta presencia se complementa no obstante con una notable variedad del resto de métodos y de técnicas (en una tendencia actual hacia la diversificación partiendo del predominio absoluto de lo métrico en los años 90 del pasado siglo), y situándose en la actualidad en este aspecto de alta variedad tras las revistas EPI y BiD.

\section{Revista general de información y documentación (RGID)}

Se analizaron 50 artículos, de los cuales un $72 \%$ fueron considerados de investigación. De ellos, sólo un $32 \%$ indicó la metodología utilizada en la investigación en el resumen del artículo. En los artículos de RGID predomina claramente la aproximación cualitativa $(63,9 \%)$.

Los temas más habituales en los artículos de investigación de $R$ GID son las fuentes de información $(23,7 \%)$ y a distancia, los estudios métricos $(13,2 \%)$ y la archivística $(13,2 \%)$, tema este último en el que destaca claramente en relación con el resto de revistas. Los datos coinciden en parte con los del estudio de López-López et al. (2001) para los años 1991-2000, en el que predominaba la temática profesional, incluyendo la archivística, seguida por tecnologías de la información y fuentes de información y en el que asimismo se señalaba el incipiente interés por los estudios métricos.

\section{$R G I D$ tiene unas características diferencia-}

les bastante marcadas, como la alta presencia de temas de archivística, metodología cualitativa e investigación histórica

La principal característica diferenciadora de $R G I D$ es que es la única publicación del sector en la que el método de investigación más utilizado es el histórico (36,1\% de sus artículos) y en clara relación con ello, las técnicas más empleadas son el análisis de contenido y la interpretación de textos. Otros métodos habituales son el descriptivo y la bibliometría y cibermetría.

Se trata de una revista con unas características diferenciales bastante marcadas, como son la presencia de temas de archivística poco habituales en otras revistas y, sobre todo, por su tendencia a la investigación cualitativa y la alta presencia de investigación histórica, aun con el contraste de una también estimable presencia de bibliometría.

\section{Scire: Representación y organización del conocimiento}

Ha publicado 56 artículos en el período analizado, algo menos del $10 \%$ del total. Tiene una alta proporción de investigación, con casi un $70 \%$ de sus artículos, y en un $40 \%$ de los mismos se presenta en el resumen información sobre la metodología utilizada. Como sucedía en RGID, predomina claramente en Scire la investigación cualitativa, en un 61,5\% de sus trabajos.

Scire se caracteriza especialmente por la importancia que otorga a la investigación teórica, lo que se manifiesta tanto en el tema de sus artículos (un $48,7 \%$ de fundamentos teóricos), como en los métodos y las técnicas de investigación mayoritarios entre su producción, que son respectivamente, el teórico o conceptual, 32,5\%, y la interpretación de textos, $36 \%$. También tienen su cabida el método descriptivo y el de diseño de sistemas de información (17,5\% en ambos casos) y la técnica del análisis de contenido (23\%).

\section{Scire muestra un perfil propio con alta presencia de investigación cualitativa, y en especial teórica}

Se trata de una publicación con un perfil propio bastante característico de alta presencia de investigación cualitativa, y en especial teórica, siendo esto último un elemento que en otras publicaciones tiene un carácter más minoritario.

\section{Conclusiones}

Este artículo presenta un análisis de siete revistas de información y documentación durante un período de tres años (2012-14) con un total de 580 artículos. Con este estudio puede concluirse a nivel general que la producción científica en este campo ha aumentado significativamente en la última década en España y las revistas también han adquirido un reconocimiento internacional en índices referentes como WoS y Scopus.

El porcentaje de artículos de investigación presenta a nivel global unos valores altos en las revistas estudiadas (68\%) y en concreto, algunas revistas destacan por su alto porcentaje, como Cyber (100\%) y REDC (91,9\%), además de que en todas menos una (BiD) el porcentaje de artículos de investigación supera al de no investigación.

Los temas más frecuentes en las revistas analizadas son las fuentes de información, los estudios métricos y las tecnologías de la información.

En cuanto a la orientación metodológica, se aprecia como las investigaciones cuantitativas y cualitativas están muy igualadas, mientras que los artículos de combinación de ambas representan, en el mejor de los casos, la décima parte del total.

En cuanto a los métodos de investigación, la investigación descriptiva es con diferencia el método más utilizado, se- 
guido por la bibliometría y cibermetría, y a continuación por la investigación teórica y la evaluativa. En el otro extremo, la investigación experimental y la bibliográfica son muy escasas.

Las técnicas de investigación más habituales son cuatro: análisis de contenido, interpretación de textos, análisis de citas o de enlaces, encuesta. En contraste, hay algunas técnicas muy poco empleadas: el grupo de discusión, la observación, el análisis de logs, el método Delphi y los experimentos. También hay que destacar que la media de técnicas utilizadas por artículo es de algo más de una.

\section{El perfil metodológico y temático dife- renciado de las revistas científicas anali- zadas permite constatar el grado de ma- durez alcanzado por la investigación en información y documentación en España}

El perfil metodológico y temático diferenciado que muestran a mediados de la segunda década del siglo XXI cada una de las revistas científicas analizadas permite constatar, aun con los déficits señalados, el grado de madurez alcanzado por la investigación en información y documentación en España, algo que también concluyeron Ríos-Hilario y Travieso-Rodríguez (2011), cuando consideraron que

"las ciencias documentales ya han alcanzado la 'mayoría de edad' y se encuentran por lo tanto en una fase de consolidación".

Futuros estudios serán necesarios -y extendiendo el análisis a otros tipos de documentos, como por ejemplo tesis doctorales- para conocer la evolución de la investigación en información y documentación, en unos momentos en que la disciplina no está exenta de potenciales transformaciones, como la fragilidad profesional del sector o la influencia creciente de las investigaciones de comunicación.

\section{Agradecimientos}

Agradecemos a Pertti Vakkari su valiosa asistencia en la preparación de la primera versión de este artículo, así como al revisor anónimo sus variadas sugerencias que han ayudado a enriquecer notablemente este trabajo.

Este estudio ha sido posible gracias a la Agència de Gestió d'Ajuts Universitaris i de Recerca (Agaur) de la Generalitat de Catalunya, así como al grupo de investigación consolidado Cultura i Continguts Digitals (2014 SGR 760).

\section{Bibliografía}

Abadal, Ernest (2006). Mètodes i tècniques de recerca en biblioteconomia i documentació, Universitat de Barcelona. http://eprints.rclis.org/15722

Abellán-García, Antonio; Serrano-Mascaraque, Esmeralda; Ernica-Lafuente, Emilio (1997). "El estado actual de la investigación en información y documentación en España, Europa, EE.UU. y algunos países iberoamericanos: proyectos de investigación sobre información, documentación y biblioteconomía entre los años 1990-1996". En: I Jornadas andaluzas de documentación. Sistemas y políticas de infor- mación en el estado de las autonomías: Situación actual y perspectivas. Sevilla, Asociación Andaluza de Documentalistas, 1997, pp. 49-59.

Amat, Carlos-Benito; Castillo-Blasco, Lourdes (1997). “¿De qué trata la documentación española?: un análisis temático de los trabajos incluidos en la base de datos ReID (Resúmenes de Información y Documentación)". Métodos de información, v. 4, n. 20, pp. 24-28.

http://www.metodosdeinformacion.es/mei/index.php/mei/ article/view/243

Asensi-Artiga, Vivina (1997). "Proyectos y líneas de investigación en relación con la tecnología de la información". Revista general de información y documentación, v. 7, n.1, pp. 47-61.

http://revistas.ucm.es/index.php/RGID/article/viewFile/ RGID9797120047A/10972

Blake, Virgil L. P. (1994). "Since Shaughnessy. Research methods in library and information science dissertations, 1975-1989". Collection management, v. 19, n. 1-2, pp. 1-42. https://doi.org/10.1300/J105v19n01_01

Busha, Charles H.; Harter, Stephen P. (1990). Métodos de investigación en bibliotecología: técnicas e interpretación. México: Universidad Nacional Autónoma de México, Centro Universitario de Investigaciones Bibliotecológicas. ISBN: 968 3616704

Cano, Virginia (1999). "Bibliometric overview of library and information science research in Spain". Journal of the American Society for Information Science, n. 50, n. 8, pp. 675-680. https://goo.gl/dMkQ8L

https://doi.org/10.1002/(SICI)1097-4571(1999)50:8<675::AIDASI5>3.0.CO;2-B

Celestino-Angulo, Sonsoles (1995). "Una reflexión sobre bibliografía profesional". En: Celestino-Angulo, Sonsoles (coord.). De libros y bibliotecas: homenaje a Rocío Caracuel. Sevilla, Universidad, pp. 95-106. ISBN: 8447202585

Cortés-Alonso, Vicenta (1993). "Cuarenta años del Boletín de Anabad: comentarios a una historia viva". En: Homenaxe a Daría Vilariño, Santiago de Compostela, Universidade, pp. 91-105. ISBN: 8471919370

Delgado-López-Cózar, Emilio (2000a). “Diagnóstico de la investigación en Biblioteconomía y Documentación en España (1976-1996): estado embrionario". Revista de investigación iberoamericana en ciencia de la información y documentación, v. 1, n. 1, pp. 79-93.

http://eprints.rclis.org/13833

Delgado-López-Cózar, Emilio (2000b). “Los métodos de investigación empleados en la literatura científica producida en Biblioteconomía y Documentación". En: I Congreso universitario de ciencias de la documentación. Teoría, historia y metodología de la documentación en España (1975-2000). Madrid: Departamento de Biblioteconomía y Documentación, Universidad Complutense, pp. 625-641.

https://pendientedemigracion.ucm.es/info/multidoc/ multidoc/revista/num10/paginas/pdfs/Edelgado.pdf

Delgado-López-Cózar, Emilio (2001). “Las revistas españolas de ciencias de la documentación: productos manifiestamen- 
te mejorables". El profesional de la información, v. 10, n. 12, pp. 46-56.

http://www.elprofesionaldelainformacion.com/contenidos/2001/ diciembre/10.pdf

Delgado-López-Cózar, Emilio (2002). La investigación en biblioteconomía y documentación. Gijón: Trea, ISBN: 84 97040414

http://ec3.ugr.es/publicaciones/Emilio_Delgado_Lopez_Cozar La_investigacion_en_Biblioteconomia_y_Documentacion_ Gijon_Trea_2002.pdf

Delgado-López-Cózar, Emilio (2003). "Las cifras de la documentación en España: 2002". El profesional de la información, v. 12, n. 5, pp. 344-367.

http://www.elprofesionaldelainformacion.com/ contenidos/2003/septiembre/1.pdf

Dimitroff, Alexandra (1995). "Research for special libraries: A quantitative analysis of the literature". Special libraries, v. 86, n. 4, pp. 256-264.

https://goo.gl/jXJXp2

Feehan, Patricia E.; Gragg, W. Lee; Havener, W. Michael; Kester, Diane D. (1987). "Library and information science research: An analysis of the 1984 journal literature". Library and information science research, v. 9, pp. 173-185.

https://goo.gl/KgXYyg

Ferran-Ferrer, Núria; Guallar, Javier; Abadal, Ernest; Server, Adán (2017). "Research methods and techniques in Spanish library and information science journals (2012-2014)". Information research, v. 22, n. 1, paper 741.

http://InformationR.net/ir/22-1/paper741.html

Frías, José-Antonio; Romero-Gómez, Purificación (1998).

"Quiénes son y qué citan los investigadores que publican en las revistas españolas de biblioteconomía y documentación". Anales de documentación, v. 1, pp. 29-53.

http://revistas.um.es/analesdoc/article/view/3101

Giménez-Toledo, Elea; Román-Román, Adelaida (2000). "Evaluación de revistas científicas: análisis comparativo de dos modelos y su aplicación a cinco revistas españolas de biblioteconomía y documentación". Interciencia, v. 25, n. 5 , pp. 234-241.

http://www.redalyc.org/articulo.oa?id=33904703

González-Alcaide, Gregorio; Alonso-Arroyo, Adolfo; Valderrama-Zurián, Juan-Carlos; Aleixandre-Benavent, Rafael (2008). "Una década de investigaciones en Anales de Documentación (1998-2007): aproximación bibliométrica y temática". Anales de documentación, v. 11, pp. 57-78. http://revistas.um.es/analesdoc/article/view/24811

Guallar, Javier (2003). "Mètodes i tècniques de recerca en els articles de documentació periodística a Espanya (19972002)". BiD: textos universitaris de biblioteconomia i documentació, n. 11.

http://bid.ub.edu/11gualla.htm

Hider, Philip; Pymm, Bob (2008). "Empirical research methods reported in high-profile LIS journal literature". Library and information science research, v. 30, n. 2, pp. 108-114. https://researchoutput.csu.edu.au/ws/portalfiles/ portal/8713857 https://doi.org/10.1016/j.lisr.2007.11.007

Järvelin, Kalervo; Vakkari, Pertti (1990). "Content analysis of research articles in library and information sciences". Library and information science research, v. 12, n. 4, pp. 395-421. https://goo.gl/bH8jKf

Järvelin, Kalervo; Vakkari, Pertti (1993). "The evolution of library and information science 1965-1985: A content analysis of journal articles". Information processing \& management, v. 29, n. 1, pp. 129-144.

https://doi.org/10.1016/0306-4573(93)90028-C

Jiménez-Hidalgo, Sonia (2007). "Análisis de la autoría en la Revista española de documentación científica (1997-2005)". Revista española de documentación científica, v. 30, n. 3, pp. 305-322.

https://doi.org/10.3989/redc.2007.v30.i3.387

Kawalec, Anna (2013). "Research trends in library and information science based on Spanish scientific publication 2000 to $2010 "$. Malaysian journal of library \& information science, v. 18, n. 2 , pp. 1-13.

http://ejum.fsktm.um.edu.my/article/1370.pdf

Krippendorff, Klaus (2004). Content analysis: an introduction to its methodology. Sage Publications Ltd. London. ISBN: 9780761915454

López-Gijón, Javier; Pérez-López, Ana; Ruiz-de-Villegasdel-Coso, Marina (1995). "Siete Jornadas bibliotecarias de Andalucía: un análisis". En: VIII Jornadas bibliotecarias de Andalucía, Huelva, Diputación Provincial, pp. 89-112. http://www.ugr.es/ aperez/doc/HUELVA.pdf

López-López, Pedro; Díaz-Hernández, Rosa-Ana; Angulo-Benítez, Sara; Giménez-Toledo, Elea (2001). “Estudio bibliométrico y de calidad de la Revista general de información y documentación (1991-2000)". Revista general de información y documentación, v. 11, n. 1. pp. 11-32.

http://revistas.ucm.es/index.php/RGID/article/viewFile/ RGID0101120011A/10314

Mochón-Bezares, Gonzalo; Sorli-Rojo, Ángela (2002). Tesauro de biblioteconomía y documentación. Madrid: ledcyt.

Neuendorf, Kimberly A. (2002). The content analysis guidebook. Thousand Oaks, CA: Sage. ISBN: 9780761919780

Ollé-Castellà, Candela; Porras, Mercè (2008). "Una mirada als vint números de BiD". BiD: textos universitaris de biblioteconomia i documentació, n. 21.

https://doi.org/10.1344/105.000000330

Pérez-Álvarez-Ossorio, José-Ramón (1997). “Cobertura temática y procedencia institucional de los artículos publicados en la Revista española de documentación científica en sus veinte años de existencia". Revista española de documentación científica, v. 20, n. 3, pp. 290-298.

https://doi.org/10.3989/redc.1997.v20.i3.584

Peritz, Bluma (1981). "The methods of library science research: some results from a bibliometric survey". Library research, v. 2, pp. 251-268.

https://goo.gl/mQZbuv

Powell, Ronald R. (1997). Basic research methods for librarians. $3^{\text {rd }}$ ed. Greenwich: Ablex. ISBN: 9781567503388 
Ríos-Hilario, Ana-Belén (1998). “Metodologías, técnicas y estrategias de investigación en las Jornadas españolas de documentación automatizada (1981-1996)". En: 6as Jornadas españolas de documentación. Los sistemas de información al servicio de la sociedad. València, 29-31 octubre 1998. València: Fesabid; Avei, pp. 735-743. ISBN: 8433146092

Ríos-Hilario, Ana-Belén (2001). “Diez años de investigación en la Revista española de documentación científica (19891999)". Revista española de documentación científica, v. 24, n. 4, pp. 433-449.

https://doi.org/10.3989/redc.2001.v24.i4.70

Ríos-Hilario, Ana-Belén; Travieso-Rodríguez, Críspulo (2013). "Estudio de la Revista española de documentación científica: análisis comparativo de los períodos 1989-1999 y 2000-2010". Revista española de documentación científica, v. 36, n. 3, e016.

https://doi.org/10.3989/redc.2013.3.990

Román-Román, Adelaida; Sorli-Rojo, Ángela (1990). “La documentación en los años 90: ¿ podemos predecir el futuro rastreando el pasado reciente de la investigación documental?". En: III Jornadas españolas de documentación automatizada, Palma de Mallorca: Universitat de les Illes Balears, v. 2, pp. 1171-1185. ISBN: 8476320868

Sánchez-Vigil, Juan-Miguel; Marcos-Recio, Juan-Carlos; Olivera-Zaldua, María (2015). "Producción científica sobre comunicación y medios en las revistas de documentación (2000-2014)". Revista española de documentación científica, v. 38, n. 4.

https://doi.org/10.3989/redc.2015.4.1257

Toumaala, Otto; Järvelin, Kalervo; Vakkari, Pertti (2014). “Evolution of library and information science, 1965-2005: Content analysis of journal articles". Jasist, v. 65, n. 7, pp. 1446-1462. https://doi.org/10.1002/asi.23034

Villagrá-Rubio, Ángel; Sorli-Rojo, Ángela (2003). "Calidad, pluralismo y prestigio de las revistas españolas de biblioteconomía y documentación". Revista española de documentación científica, v. 26, n. 4, pp. 445-460.

https://doi.org/10.3989/redc.2003.v26.i4.145

\section{Anexos}

\section{Categorías temáticas}

\begin{tabular}{|c|c|}
\hline Categoría & Subcategoría (descriptores) \\
\hline $\begin{array}{l}\text { Acceso y recuperación } \\
\text { de la información }\end{array}$ & $\begin{array}{l}\text { Derechos de autor, datos abiertos, acceso abierto, administración electrónica, recuperación de la información, posiciona- } \\
\text { miento web, datos masivos. }\end{array}$ \\
\hline Archivística & Gestión documental, descripción archivística, fondo de archivos, evaluación documental. \\
\hline Comunicación & $\begin{array}{l}\text { Medios de comunicación, redes sociales, comunicación científica, comunicación audiovisual, publicidad, comunicación } \\
\text { empresarial }\end{array}$ \\
\hline Estudios métricos & Bibliometría, cienciometría, producción bibliográfica, altmétricas, analítica web. \\
\hline $\begin{array}{l}\text { Fundamentos teóricos } \\
\text { y aspectos generales }\end{array}$ & $\begin{array}{l}\text { Biblioteconomía, documentación, documentación periodística, organización y representación del conocimiento, tesauro, } \\
\text { ontologías, clasificaciones, terminología, gestión de la información y del conocimiento, vigilancia tecnológica, inteligencia } \\
\text { competitiva, sociedad de la información, análisis de contenido. }\end{array}$ \\
\hline $\begin{array}{l}\text { Fuentes y recursos de } \\
\text { información }\end{array}$ & $\begin{array}{l}\text { Bibliotecas digitales, repositorios, portales web, publicaciones en serie, internet, industria editorial, industria audiovisual, } \\
\text { hemerotecas digitales, directorios, catálogos, bibliografía, bases de datos, comercio de libros, evaluación de webs, libros } \\
\text { electrónicos, recursos electrónicos, planos, revistas, fotografías, carteles, imágenes, patrimonio bibliográfico, tarjetas pos- } \\
\text { tales, blogs, mapas, archivos audiovisuales, tesis doctorales, patentes, libros antiguos, encuadernaciones, libros de artista. }\end{array}$ \\
\hline Proceso técnico & Catalogación, indexación, clasificación, preservación y conservación, análisis documental, gestión de colecciones. \\
\hline Profesionales & $\begin{array}{l}\text { Competencias profesionales, formación, centros de enseñanza, perfil profesional, situación laboral, mercado de trabajo, } \\
\text { biografías, ética. }\end{array}$ \\
\hline $\begin{array}{l}\text { Técnicas auxiliares y } \\
\text { otras disciplinas }\end{array}$ & $\begin{array}{l}\text { Historia, historiografía, historia de la imprenta, historia del libro, derecho, propiedad intelectual, protección de datos, filo- } \\
\text { sofía, política, políticas de información, economía, enseñanza, métodos y técnicas de aprendizaje, marketing, mecenazgo, } \\
\text { humanidades, biotecnología, terapéutica, epígrafe, genealogía, paleografía, investigación científica, publicación científica, } \\
\text { museología, normalización, técnicas y sistemas de gestión, evaluación científica, numismática, cine, sociología, ciencias de la } \\
\text { salud, pintura, química, música, fotografía, educación física, empresariales, empresas de servicios documentales. }\end{array}$ \\
\hline $\begin{array}{l}\text { Tecnologías de la } \\
\text { información }\end{array}$ & $\begin{array}{l}\text { Informática, software y aplicaciones informáticas, preservación digital, web semántica, accesibilidad, digitalización, web } \\
\text { 2.0, web móvil, usabilidad, diseño de webs, metadatos, web social, visualización de la información, realidad aumentada. }\end{array}$ \\
\hline $\begin{array}{l}\text { Unidades de } \\
\text { información y } \\
\text { servicios }\end{array}$ & $\begin{array}{l}\text { Bibliotecas, bibliotecas públicas, bibliotecas universitarias, bibliotecas especializadas, bibliotecas escolares, bibliotecas } \\
\text { nacionales, archivos, archivos audiovisuales, museos, centros de documentación, cooperación bibliotecaria, promoción } \\
\text { de la lectura, cartotecas, servicios, servicio de extensión bibliotecaria, servicio de préstamo, servicios de información, eva- } \\
\text { luación de los servicios. }\end{array}$ \\
\hline Usuarios & Estudios de usuarios, formación de usuarios, alfabetización informacional. \\
\hline
\end{tabular}




\section{Métodos de investigación}

\begin{tabular}{|c|c|}
\hline Método & Descripción \\
\hline $\begin{array}{l}\text { Investigación } \\
\text { descriptiva }\end{array}$ & $\begin{array}{l}\text { Se utiliza para describir el estado de la cuestión de fenómenos poniendo de manifiesto una serie de variables y condicio- } \\
\text { nes. Su objeto es la descripción de fenómenos, quiere conocer cómo es la realidad. Intenta estudiar el presente mediante } \\
\text { la descripción de lo que ahora existe. }\end{array}$ \\
\hline $\begin{array}{l}\text { Investigación } \\
\text { bibliométrica y } \\
\text { cibermétrica }\end{array}$ & $\begin{array}{l}\text { Aplicación de métodos matemáticos y estadísticos en el estudio de la ciencia y de la producción científica (crecimiento, } \\
\text { envejecimiento, dispersión), los autores que la producen (productividad, visibilidad, impacto, etc.) y también a su uso; así } \\
\text { como estudios métricos sobre la información digital y la web. }\end{array}$ \\
\hline Investigación histórica & $\begin{array}{l}\text { Estudios que persiguen describir y comprender hechos pasados y experiencias humanas por medio de la recogida, eva- } \\
\text { luación, análisis e interpretación de datos históricos. }\end{array}$ \\
\hline $\begin{array}{l}\text { Investigación } \\
\text { bibliográfica }\end{array}$ & $\begin{array}{l}\text { Hace referencia a los estudios bibliográficos descriptivos que evalúan los libros, sus propiedades físicas, publicaciones, } \\
\text { impresión, etc. También incluye bibliografías sistemáticas como bibliografías de autor o temáticas. }\end{array}$ \\
\hline $\begin{array}{l}\text { Investigación } \\
\text { experimental }\end{array}$ & Investigación que quiere establecer una relación causal entre diversas variables. \\
\hline $\begin{array}{l}\text { Investigación } \\
\text { evaluativa }\end{array}$ & $\begin{array}{l}\text { Tipo de investigación aplicada que tiene como objetivo juzgar el valor, utilidad, funcionalidad, etc. de los programas/ } \\
\text { sistemas de acuerdo con unos determinados criterios. }\end{array}$ \\
\hline $\begin{array}{l}\text { Investigación teórica } \\
\text { o conceptual }\end{array}$ & Desarrollo de modelos conceptuales o marcos teóricos por medio de la reflexión y el análisis lógico. \\
\hline $\begin{array}{l}\text { Diseño de sistemas de } \\
\text { información }\end{array}$ & $\begin{array}{l}\text { Incluye aquellos estudios que se ocupan del análisis y diseño de sistemas de información y del desarrollo de herramientas } \\
\text { de recuperación y de almacenamiento de la información. }\end{array}$ \\
\hline Revisión de literatura & Estudios que presentan un análisis detallado de las publicaciones existentes sobre un tema. \\
\hline
\end{tabular}

3. Técnicas de investigación

\begin{tabular}{|c|c|}
\hline Técnica & Descripción \\
\hline $\begin{array}{l}\text { Análisis de citas o } \\
\text { de enlaces }\end{array}$ & $\begin{array}{l}\text { Recuento y estudio de las referencias bibliográficas que han sido citadas en un trabajo científico (en bibliometría); recuento } \\
\text { y estudio de enlaces web (en cibermetría). }\end{array}$ \\
\hline $\begin{array}{l}\text { Análisis de } \\
\text { contenido }\end{array}$ & $\begin{array}{l}\text { Estudios objetivos y cuantitativos de documentos y otras formas de comunicación que examinan la presencia y frecuencia } \\
\text { de patrones de palabras, frases, conceptos, etc. (Powell, 1997). Utilizado especialmente para estudios de carácter lingüístico, } \\
\text { semiótico, de comunicación social. }\end{array}$ \\
\hline $\begin{array}{l}\text { Análisis secundario } \\
\text { (de datos existentes) }\end{array}$ & $\begin{array}{l}\text { Se llevan a cabo análisis de datos e informaciones ya publicadas para dibujar un contexto que proporcione nueva informa- } \\
\text { ción. Se recopila información ya preparada para reutilizarla. }\end{array}$ \\
\hline $\begin{array}{l}\text { Análisis } \\
\text { transaccional (logs) }\end{array}$ & $\begin{array}{l}\text { Estudio de las acciones realizadas por el usuario en su interacción con un sistema informático (p. ej. un SRI), a partir de su } \\
\text { registro en un archivo. }\end{array}$ \\
\hline $\begin{array}{l}\text { Análisis y diseño } \\
\text { de sistemas de } \\
\text { información }\end{array}$ & $\begin{array}{l}\text { Estudios evaluativos, comparativos o de desarrollo de productos, servicios o sistemas de información. Tienen un carácter } \\
\text { marcadamente técnico y aplicado. }\end{array}$ \\
\hline $\begin{array}{l}\text { Encuesta/ } \\
\text { Cuestionario }\end{array}$ & $\begin{array}{l}\text { Instrumento estructurado para la recogida de datos primarios a grupos de personas. El objetivo es determinar los conoci- } \\
\text { mientos, opiniones o actitudes de los encuestados sobre diversos aspectos. }\end{array}$ \\
\hline Entrevista & $\begin{array}{l}\text { Se utiliza la comunicación verbal para obtener información sobre un tema definido. Metodológicamente muy similar a los } \\
\text { cuestionarios. Diferencia: las respuestas no son escritas por el interrogado, sino por el encuestador, que hace las preguntas } \\
\text { personalmente. }\end{array}$ \\
\hline Estudio de caso & $\begin{array}{l}\text { Análisis y descripciones detalladas de uno o pocos individuos u organizaciones, que se consideran representativos del con- } \\
\text { junto del que forman parte. }\end{array}$ \\
\hline Estudio Delphi & $\begin{array}{l}\text { Preguntas individuales secuenciales, normalmente utilizando un cuestionario, retroalimentadas con información y opinión } \\
\text { interpuesta. Procedimiento pensado para generar opiniones consensuadas mediante varios intercambios de cuestionario } \\
\text { ("vueltas") que permiten que los expertos puedan ir modificando sus respuestas a partir del conocimiento de la opinión de } \\
\text { otros expertos. }\end{array}$ \\
\hline $\begin{array}{l}\text { Interpretación de } \\
\text { textos }\end{array}$ & $\begin{array}{l}\text { Análisis y lectura de textos con el fin de extraer conclusiones sobre eventos, mentalidades, etc. Se utiliza especialmente en } \\
\text { estudios históricos, filosóficos, etc., o en general en aquellos que analizan el pensamiento de los autores. }\end{array}$ \\
\hline Experimento & $\begin{array}{l}\text { Se prepara una situación de laboratorio en la cual se manipulan los valores de una o más variables y se observan los efectos } \\
\text { en otras variables (denominadas dependientes) }\end{array}$ \\
\hline Grupo de discusión & $\begin{array}{l}\text { Encuentros sucesivos de pequeños grupos de personas que se organizan para explorar sus experiencias y puntos de vista } \\
\text { sobre un determinado conjunto de aspectos. }\end{array}$ \\
\hline Observación & Vigilancia directa y grabación de las dimensiones del fenómeno que se estudia. \\
\hline
\end{tabular}

